\title{
Reconsidering postpartum weight loss counselling interventions. (First comment on BJOG-19-1869)
}

\author{
Debra Bick ${ }^{1}$, Cath Taylor ${ }^{2}$, and Michael Ussher ${ }^{3}$ \\ ${ }^{1}$ University of Warwick \\ ${ }^{2}$ University of Surrey \\ ${ }^{3} \mathrm{St}$ Georges University of London
}

May 5, 2020

Letter to the Editor, BJOG Exchange

\section{Re: Reconsidering postpartum weight loss counselling interventions}

Dear Editor

We were interested in the commentary on our study ${ }^{1},{ }^{2}$ but concerned about how our work was interpreted.

It is inaccurate to refer to our intervention as 'counselling'. The commercial groups offered to women are interactive, underpinned by behaviour change and social cognitive theory. Groups focus on enhancing and sustaining motivation and self-efficacy for weight management, through goal setting, self-monitoring, and positive reinforcement. The programme focuses on long-term lifestyle changes, to counter factors that impact on an individual's weight management.

Many previous trials focused on pregnancy interventions with postnatal follow-up, rather than specific postnatal intervention. A review of lifestyle interventions in overweight and obese pregnant or postpartum women for postnatal weight management found insufficient evidence to conclude if pregnancy interventions were effective. In contrast, postpartum weight management was achievable with intensive lifestyle interventions commencing postnatally, although further evidence is needed ${ }^{3}$.

Reference is made to only $17 \%$ of women accepting our recruitment invitation, our uptake similar to that reported in a pregnancy weight management trial conducted in similar real-world UK settings ${ }^{4}$. We recruited women from an inner-city unit in one of the most ethnically and economically diverse populations in the UK. The majority approached did not have the opportunity to consider participation, as women could not be traced or did not respond to calls. Only two women offered a recruitment appointment later declined. Our high response to follow-up (>80\% both trial groups) should also be noted.

We collected in-depth data on barriers to attendance to weight management groups, which showed for most women it was lack of opportunity (lack of time/fit with routines), rather than 'difficult' access. We are confident that advising women they can attend any group at any time will increase uptake in a future trial.

As Bodnar ${ }^{1}$ highlights, only two trials to date used web-based or mobile interventions in postpartum weight management studies. We undertook considerable planning work with local women, some of whom had previously used online resources in an effort to manage postpartum weight. They were clear that women wanted face to face support in group settings, where they could meet other people. Very few women in our trial used web-based or mobile interventions (only 6 control and 2 intervention women when asked at 12 months postnatally). Our service user group ensured all communication with women reflected sensitive and appropriate language. 
We agree that the timing of the intervention may not suit all women. However, evidence of an optimal time to commence an intervention was not available. Our findings support extending duration of offer in a future trial, as most control women who commenced some form of weight management support did so around 5 6 months postnatally. While we found higher depression scores among intervention women, this should be treated with caution and is an outcome of importance going forward.

Our findings show that a future definitive trial of clinical and cost effectiveness of group based commercial weight management support for postnatal women in the UK with higher BMIs at pregnancy commencement remains an important consideration.

Yours faithfully

Debra Bick, ${ }^{1}$ Cath Taylor, ${ }^{2}$ Michael Ussher ${ }^{3}$

1) Warwick Clinical Trials Unit, University of Warwick

2) School of Health Sciences, University of Surrey

3) Population Health Research Institute, St George's, University of London and Institute for Social Marketing and Health, University of Stirling

On behalf of the SWAN Trial Team.

\section{REFERENCES}

1. Bodnar LM. Reconsidering postpartum weight loss counselling interventions. Mini commentary. BJOG. 2019 Dec 22. doi: 10.1111/1471-0528.16069

2. Bick D, Taylor C, Bhavnani V, Healey A, Seed P, Roberts S, Zasada M, Avery A, Craig V, Khazaezadah N, McMullen S, O'Connor S, Oki B, Oteng Ntim E, Poston L, Ussher M. Lifestyle information and commercial weight management groups to support maternal postnatal weight management and positive lifestyle behaviour: the SWAN feasibility randomised controlled trial. BJOG. 2019 Dec 5. doi: 10.1111/1471-0528.16043. [Epub ahead of print]

3. Dalrymple KV, Flynn AC, Relph SA, O'Keeffe M, Poston L. Nutrients. 2018 Nov 7;10(11). pii: E1704. doi: 10.3390/nu10111704.

4. Poston L, Bell R, Croker H, Flynn AC, Godfrey KM, Goff L et al. Effect of a behavioural intervention in obese pregnant women (the UPBEAT study): a multi-centre, randomised controlled trial. Lancet Diab Endocrinol 2015; 3: 767-77 